\title{
Nanoparticle-Templated Self-Assembly Dynamics of Viral Capsids Probed by UV-Vis Absorbance Spectroscopy and X-Ray Scattering
}

\author{
Andrew Burke, Maelenn Chevreuil, Alisier Paris, Vanessa Delagrange, \\ Claire Goldmann, Doru Constantin, ${ }^{*}$ and Guillaume Tresset ${ }^{\dagger}$ \\ Laboratoire de Physique des Solides, CNRS, Univ. Paris-Sud, \\ Université Paris-Saclay, 91405 Orsay Cedex, France \\ Javier Pérez \\ SOLEIL synchrotron, 91192 Gif-sur-Yvette Cedex, France
}

(Dated: October 4, 2018)

\begin{abstract}
Viral capsid proteins have the remarkable ability to self-assemble around a cargo core, whether their genome, a polyelectrolyte or an inorganic nanoparticle. Although the equilibrium properties of such composite objects have been broadly investigated, little is known about the kinetic pathways leading to their assembly. By exploiting the plasmon resonance effect of gold nanoparticles, we show that the adsorption of viral proteins can be monitored through the redshift of the resonance wavelength. Kinetic measurements reveal that the process occurs in less than a second. The spatial arrangement of nanoparticles is probed by time-resolved small-angle X-ray scattering, and we observe the formation of transient aggregates with a lifetime in the hour range. The biocompatibility of gold nanoparticles induced by adsorbed proteins is of great importance for their use in biomedicine and the reported results should help better understand the processes of formation.
\end{abstract}

\section{INTRODUCTION}

The emerging study of biomolecular assemblies is an ${ }^{52}$ area of great fascination due to the astounding precision ${ }^{53}$ with which nanometer-sized objects are able to sponta- ${ }^{54}$ neously assemble without any external intervention [1]. 55 The assembly of viral proteins to form a capsid - i.e., the ${ }^{50}$ protein shell of a virus - is one such process [2-8]. Un- 57 derstanding the underlying mechanisms is fundamental 58 in transferring the principles onto synthetic and macro- 59 scopic materials. A recent point of intrigue concerns how 60 the self-assembly of viral proteins occurs around another ${ }^{61}$ material, e.g., the viral genome [9-18], a synthetic poly- 62 mer [19-24] or a nanoparticle [25-33].

Gold nanoparticles (AuNPs) are of particular interest ${ }^{64}$ because they hold a lot of potential applications in pho- 65 tonics, electronics and biomedicine [34]. Once materials 66 are reduced to nanometer sizes, a variety of interesting ${ }^{67}$ properties emerge, which are not seen in bulk materials. 68 These distinctive optical and electromagnetic properties 69 can be modified by changing parameters such as the size, 70 shape and surface charge density of the nanoparticle in ${ }^{71}$ order to tune them for a particular function [35]. The ${ }^{72}$ existence of the surface plasmon resonance at the in- 73 terface between a noble metal nanoparticle and the sur- 74 rounding medium, serves to enhance the electromagnetic ${ }^{75}$ properties, notably absorption and scattering, in a way 76 which is characteristic to each type of metal nanopar- 77 ticle [36]. For biomedical applications in particular, it 78 is crucial that nanoparticles are well stabilized because ${ }^{79}$ aggregation prevents them from diffusing through the tis- 80

\footnotetext{
*doru.constantin@u-psud.fr

$\dagger$ guillaume.tresset@u-psud.fr
}

sues and shortens their circulatory lifetime. Within this framework, the capsid formed by viral proteins around a synthetic core constitutes an efficient and biocompatible stabilizing agent as well as a protective barrier for the body.

The simplest viruses are made up of a capsid protecting the genome in the form of nucleic acids. Half of the viruses on Earth display an icosahedral symmetry and the capsid proteins are therefore arrayed onto an icosahedral lattice forming a two-dimensional crystal structure [37-39]. For example, brome mosaic virus (BMV) and cowpea chlorotic mottle virus (CCMV) are both single-stranded RNA viruses infecting plants, and their icosahedral capsid comprises 90 dimeric subunits that can self-assemble in vitro depending upon ionic conditions. The proteins interact with each other through a short-range hydrophobic and/or van der Waals attraction and a long-range electrostatic repulsion [40-42], while the subunit-genome, or subunit-cargo, interaction is realized by an electrostatic attraction. Daniel and coworkers [43] showed that the higher the surface charge density of ligand-stabilized AuNPs, the better their encapsulation into capsids derived from BMV. Theoretical investigations demonstrated that a threshold surface charge density must be exceeded and that the encapsulation efficiency is gradual due to charge regulation and size polydispersity of the nanoparticles [31]. He and coworkers [29] observed that the capsid of hepatitis B virus can withstand an increase of the nanoparticle diameter or adapt to it by generating packing defects.

While the equilibrium properties of empty or filled viral capsids have been thoroughly studied, the associated kinetic pathways, in contrast, are still poorly understood. Time-course static light scattering [44, 45], resistive-pulse sensing through nanofluidic channel [46], atomic force microscopy [47, 48], nuclear magnetic reso- 
nance [49], charge detection mass spectrometry [50] and $_{137}$ time-resolved small-angle X-ray scattering (TR-SAXS) [51-55] have been used to shed some light on the self-139 assembly dynamics of empty or nucleic acids-filled cap-140 sids. The dynamics of AuNPs encapsulation is chal-141 lenging to probe, because the adsorption of capsid pro-142 teins occurs very rapidly and, consequently, techniques 143 with long acquisition times are ineffective. Malyutin and ${ }_{144}$ Dragnea [30] carried out a kinetic study by time-course light scattering and cryo-electron tomography on the encapsulation of AuNPs into BMV capsids. They reported a two-step mechanism where aggregates of nanoparticles ${ }^{145}$ are transiently formed upon the presence of proteins, then stable capsids enclosing nanoparticles bud off the ${ }^{146}$ aggregates.

In this work, we exploit the plasmon resonance effect $\mathrm{t}^{148}$ of AuNPs to probe the adsorption of CCMV capsid pro- ${ }^{149}$ teins on ligand-stabilized AuNPs. We also study the ag- ${ }^{150}$ gregation mechanism proposed by Malyutin and Dragnea ${ }^{151}$ with TR-SAXS and we clearly detect the formation of ag- ${ }^{152}$ gregates, which are subsequently dissociated over a long ${ }^{153}$ timescale. We expect this study to promote further inves- ${ }^{154}$ tigations on the self-assembly dynamics of nanoparticle-155 templated viral capsids and to help design systems useful156 to biomedical and imaging applications.

\section{EXPERIMENTAL METHODS}

\section{A. CCMV virion purification}

Cowpea chlorotic mottle virus (CCMV) is purified ${ }^{164}$ from infected blackeye cowpea leaves (Vigna ungiculata) ${ }^{165}$ following a protocol developed by Ali and Roossinck [56]. ${ }^{160}$ $140 \mathrm{~g}$ of infected leaves are homogenized with $300 \mathrm{~mL}$ of ${ }^{167}$ $0.15 \mathrm{M}$ sodium acetate $p \mathrm{H} 4.8$ in a blender, then 300 $\mathrm{mL}$ of ice-cold chloroform are added and the solution is stirred for $10 \mathrm{~min}$. After $10 \mathrm{~min}$ of centrifugation at168 $10000 \times g$, the virions are precipitated by stirring the aqueous upper phase for $30 \mathrm{~min}$ in $20 \mathrm{mM} \mathrm{NaCl}$ and $8 \%_{169}$ of poly(ethylene glycol) (MW 8000). After another cen- ${ }_{170}$ trifugation at $10000 \times g$ for $10 \mathrm{~min}$, the pellet is dissolved ${ }_{171}$ in $21 \mathrm{~mL}$ of $50 \mathrm{mM}$ sodium acetate $p \mathrm{H} 4.8$ and stirred $_{172}$ for $1 \mathrm{~h}$. The solution is centrifuged at $8000 \times g$ for $10_{173}$ min and the supernatant is centrifuged through a $20 \%_{174}$ $(\mathrm{w} / \mathrm{v})$ sucrose cushion at $150000 \times g$ for $2 \mathrm{~h}$. The virions ${ }_{175}$ are collected in the pellet and stored in $50 \mathrm{mM}$ sodium $_{176}$ acetate $p \mathrm{H} 4.8$ at $-80{ }^{\circ} \mathrm{C}$ until use. Samples are deemed ${ }_{177}$ pure when the extinction ratio verifies $A_{260} / A_{280}>1.60 ._{178}$

\section{B. Capsid protein purification}

$10 \mathrm{mg}$ of purified virions are dissociated by a 24-183 $\mathrm{h}$ dialysis against $0.5 \mathrm{M} \mathrm{CaCl} 2,1 \mathrm{mM}$ ethylenedi-184

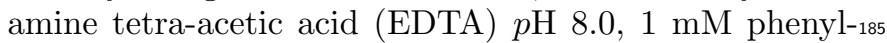
methylsulfonyl fluoride, $1 \mathrm{mM}$ dithiothreitol, $50 \mathrm{mM}_{186}$ tris(hydroxymethyl)aminomethane (Tris)-HCl $p \mathrm{H} \quad 7.5 .187$
After $18 \mathrm{~h}$ of centrifugation at $150000 \times g, 1-\mathrm{mL}$ aliquots of the supernatant are extracted and conserved if $A_{280} / A_{260}>1.65$. The capsid proteins are kept in their assembled form in $0.5 \mathrm{M} \mathrm{NaCl}, 1 \mathrm{mM}$ EDTA $p \mathrm{H}$ 8.0, 50 mM sodium acetate $p \mathrm{H} 4.8$ at $4{ }^{\circ} \mathrm{C}$ until use within two weeks after purification. The protein concentration is estimated with an extinction coefficient $\epsilon_{\text {percent }}=8.34$ at $280 \mathrm{~nm}$.

\section{Gold nanoparticle synthesis}

We use three batches of gold nanoparticles (AuNPs), synthesized by different methods: Most UV-Vis absorbance experiments are done with AuNPs synthesized in toluene using oleylamine as a capping agent [57]. We study the effect of ligand charge (see Figure 3 ) on particles obtained by a seed-growth method in aqueous solution [58]. Particles used in TR-SAXS experiments (see Figure 5) are synthesized via an improved citrate method [59]. Particle sizes are similar in all batches.

Two types of ligands, similar to those used by Daniel et al. [43], are used to functionalize the AuNPs: a nonionic thiolated surfactant, (1-mercaptoundec-11yl)hexa(ethylene glycol), $\mathrm{HS}_{-} \mathrm{C}_{11}-\mathrm{EG}_{6}-\mathrm{OH}$, denoted as MUDOL and its acidic (carboxylated) counterpart, HS$\mathrm{C}_{11}-\mathrm{EG}_{6}-\mathrm{CH}_{2} \mathrm{COOH}$, denoted as $\mathrm{EG}_{6}-\mathrm{COOH}$. The latter is purchased from ProChimia Surfaces (Poland). The AuNPs synthesized in water, initially stabilized by 0.5 $\mathrm{mM}$ CTAB, are incubated overnight with an excess of either ligand. Those synthesized in toluene are resuspended in THF, functionalized and then transferred to water. They are afterwards washed in pure water and stored at room temperature.

\section{Encapsulation of AuNPs into viral capsids}

CCMV capsid proteins in the form of dimeric subunits are initially dispersed in $0.5 \mathrm{M} \mathrm{NaCl}, 1 \mathrm{mM}$ EDTA $p \mathrm{H}$

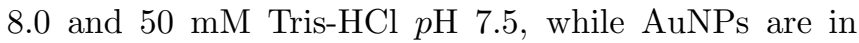
pure water. For transmission electron microscopy and static extinction spectroscopy, subunits and AuNPs are dialyzed together against $50 \mathrm{mM} \mathrm{NaCl}, 1 \mathrm{mM}$ EDTA $p \mathrm{H}$ 8.0 and $50 \mathrm{mM}$ sodium acetate $p \mathrm{H} 4.8$. Aniagyei et al. [27] found that acidic $p \mathrm{H}$ renders the adsorbed CCMV protein layer more compact at the surface of AuNPs than at neutral $p \mathrm{H}$. However, $\mathrm{EG}_{6}-\mathrm{COOH}$-stabilized AuNPs are not fully stable at low $p \mathrm{H}$. As a consequence, our attempts to carry out kinetics by mixing rapidly subunits and AuNPs in acidic buffer failed because the AuNPs precipitated before being stabilized further by the subunits. Therefore, adsorption kinetics and TR-SAXS experiments are performed by mixing subunits, AuNPs and pure water in such a way that the final ionic strength is about $0.1 \mathrm{M}$ and the $p \mathrm{H}$ remains at 7.5. In all experiments, the final protein concentration is within 0.1-0.3 
g. $\mathrm{L}^{-1}$ and the subunit-to-AuNP molar ratio ranges from 231 180:1 to $3600: 1$ so that proteins are in large excess.

\section{E. Transmission electron microscopy}

$5 \mu \mathrm{L}$ of sample solution is placed during $10 \mathrm{~min}$ on a carbon grid treated by glow discharge, and blotted with the edge of a filter paper. A drop of $1 \%(\mathrm{v} / \mathrm{v})$ ammonium molybdate is deposited on the grid for $30 \mathrm{~s}$, then removed with filter paper, and the grid is allowed to air dry for at least $10 \mathrm{~min}$. Transmission electron microscopy (TEM) is performed with a JEOL JEM-2010 microscope equipped with a $200-\mathrm{kV}$ field emission gun. The samples are imaged with magnifications comprised between $\times 20000$ and $\times 50000$ and the images are collected with a Gatan Ultrascan 4K CCD camera at a defocus comprised between 1.5 and $3 \mu \mathrm{m}$.

\section{F. UV-Vis absorbance spectroscopy}

For static experiments, we measure the absorbance spectrum of dilute particles for wavelengths comprised between 400 and $1400 \mathrm{~nm}$ with an Agilent Cary 5000 spectrometer. Samples are placed in polystyrene cuvettes with a $10-\mathrm{mm}$ optical path.

For adsorption kinetics, we use a Biologic SFM-3000 stopped-flow apparatus (France) equipped with a manual monochromator and a high sensitivity photomultiplier. The wavelength is set to $530 \mathrm{~nm}$ and the extinction is collected with a fixed time interval of $10 \mathrm{~ms}$.

\section{G. Time-resolved small-angle X-ray scattering}

X-ray scattering measurements are performed with thero SWING beamline at the SOLEIL synchrotron facility 241 (Gif-sur-Yvette, France). The wavelength is set to $\lambda=1_{242}$ $\AA$ and the sample-to-detector distance is $4 \mathrm{~m}$, which pro-243 vides scattering wavenumbers $q(q=4 \pi / \lambda \sin (\theta / 2)$ where 244 $\theta$ is the scattering angle) ranging from $2.3 \times 10^{-3}$ to $0.28_{245}$ $\AA^{-1}$. Two-dimensional scattering images are recorded 246 on an AVIEX large area CCD detector with an exposure 247 time of $250 \mathrm{~ms}$. The rapid mixing of AuNPs with sub-248 units is carried out with a Biologic SFM-300 stopped-flow 249 apparatus (France) equipped with three syringes and the 250 flow is stabilized by a hard stop valve. The scattering 251 intensities are converted into absolute units after sub-252 tracting the contribution of the buffer, and the uncer-253 tainties are calculated after circular averaging using the 254 FOXTROT software package.

\section{RESULTS}

\section{A. Morphology of protein-coated AuNPs observed by electron microscopy}

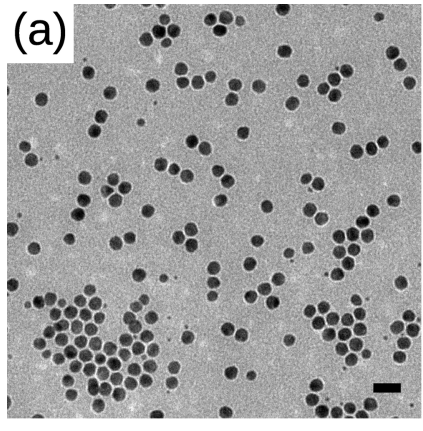

(c)

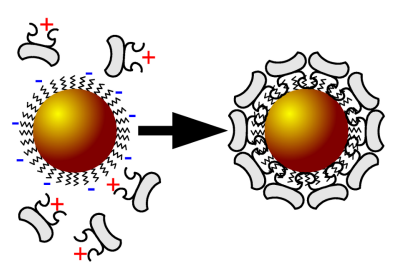

(b)

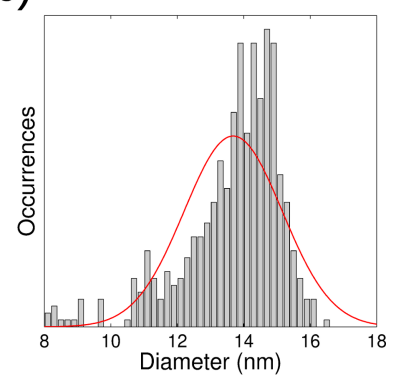

(d)

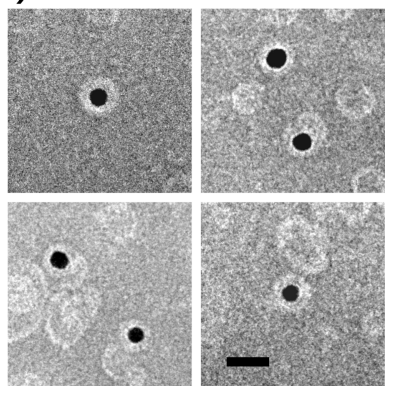

FIG. 1. Transmission electron microscopy of naked and protein-coated EG$_{6}-\mathrm{COOH}$-stabilized AuNPs. (a) Naked AuNPs and (b) their size distribution inferred from TEM images. (c) Schematics depicting the coating of ligand-stabilized AuNPs by subunits. (d) Protein-coated AuNPs. Scale bars are $30 \mathrm{~nm}$.

Figure 1(a) shows a TEM image of naked $\mathrm{EG}_{6}-\mathrm{COOH}-$ stabilized AuNPs in pure water after synthesis. Note that all AuNPs used in this study are ligand-stabilized and naked AuNPs refers hereafter to as ligand-stabilized AuNPs without adsorbed proteins. Image analysis gives a diameter distribution of $13.7 \pm 1.5$ (mean \pm s.d.) $\mathrm{nm}$ [Fig. 1(b)], that is, the AuNPs are fairly monodisperse. At neutral $p \mathrm{H}$ and high ionic strength $(0.5 \mathrm{M})$, CCMV capsid proteins are in the form of dimeric subunits. Each subunit carries two flexible, cationic arms rich in arginine. When $\mathrm{EG}_{6}-\mathrm{COOH}$-stabilized AuNPs and subunits are mixed at neutral $p \mathrm{H}$ and low ionic strength $(\sim 0.1$ $\mathrm{M})$, the cationic arms are electrostatically attracted by the anionic moiety of the ligands. As a result, the subunits are adsorbed at the surface of the nanoparticles and form a capsid with some defects [Fig. 1(c)]; when the $p \mathrm{H}$ becomes acidic, the capsids are consolidated. Figure 1(d) depicts such AuNPs encapsulated into viral capsids. The size of the capsids is close to that of the native virions, i.e., $28 \mathrm{~nm}$, and since the subunits are in excess, several empty capsids or pieces of capsids are coexisting with the protein-coated AuNPs. 
B. Redshift of plasmon resonance induced by capsid proteins

$$
\alpha=3 V \frac{\epsilon_{\mathrm{Au}}-\epsilon_{\mathrm{W}}}{\epsilon_{\mathrm{Au}}+2 \epsilon_{\mathrm{W}}}
$$

A homogeneous nano-sized gold sphere embedded in a263 uniform electric field has a polarizability $\alpha$ (defined as the 264 ratio between the induced dipole moment of the sphere265 and the applied electric field) given by [60]: where $V$ is the volume of the sphere, $\epsilon_{\mathrm{Au}}$ is the relative permittivity of gold and $\epsilon_{\mathrm{W}}$ is the relative permittivity of the medium, here water. If, however, we consider a spherical AuNP coated by a shell layer with a different dielectric constant, the polarizability becomes [60]:

$$
\alpha=3 V \frac{\left(\epsilon_{\mathrm{S}}-\epsilon_{\mathrm{W}}\right)\left(\epsilon_{\mathrm{Au}}+2 \epsilon_{\mathrm{S}}\right)+f\left(2 \epsilon_{\mathrm{S}}+\epsilon_{\mathrm{W}}\right)\left(\epsilon_{\mathrm{Au}}-\epsilon_{\mathrm{S}}\right)}{\left(\epsilon_{\mathrm{Au}}+2 \epsilon_{\mathrm{S}}\right)\left(\epsilon_{\mathrm{S}}+2 \epsilon_{\mathrm{W}}\right)+2 f\left(\epsilon_{\mathrm{S}}-\epsilon_{\mathrm{W}}\right)\left(\epsilon_{\mathrm{Au}}-\epsilon_{\mathrm{S}}\right)}
$$

where $f=[R /(R+d)]^{3}$ with $R$ the radius of the AuNP and $d$ the shell thickness. $\epsilon_{\mathrm{S}}$ denotes the relative permittivity of the shell layer.

Changes in $\alpha$ can be probed using UV-Vis absorbance spectroscopy, since the beam extinction $E$ is proportional to the extinction cross-section: $E=N \sigma_{\text {ext }} l / \ln (10)$, where $N$ is the number of nanoparticles per unit volume and $l$ is the length of optical path. The extinction crosssection $\sigma_{\text {ext }}$ is the sum of the contributions arising from absorption and scattering. The scattering contribution is here negligible due to the small size of the nanoparticles. Therefore, $\sigma_{\text {ext }}$ is dominated by the absorption cross section $\sigma_{\text {abs }}$ :

$$
\sigma_{\mathrm{ext}} \approx \sigma_{\mathrm{abs}}=\frac{2 \pi}{\lambda_{\mathrm{W}}} \Im(\alpha)
$$

with $\lambda_{\mathrm{W}}$ the wavelength of the incident light in water and $\Im(\alpha)$ the imaginary part of $\alpha$. Despite its simplicity, the model is quite accurate and allows extracting reliable parameters via full-curve fitting of the extinction curves [61].

Figure 2(a) gives the calculated absorption cross sections [Eq. (2)] of AuNPs coated with a dielectric shell. Note that the refractive index $n$ is related to the relative permittivity $\epsilon$ by $\epsilon=n^{2}$. $\epsilon_{\mathrm{Au}}$ is a complex function of the wavelength and is derived from an analytical model (see Appendix). In the absence of a shell, i.e., when $n_{\mathrm{S}}=n_{\mathrm{W}}$, the absorption cross-section exhibits a plasmon resonance peak at a wavelength around $520 \mathrm{~nm}$. As the refractive index of the shell increases, the peak shifts to higher values and the maximal amplitude increases. The shift of wavelength between the spectrum with no shell and that with $n_{\mathrm{S}}=1.4$ is $3 \mathrm{~nm}$, and amounts to $8 \mathrm{~nm}$ when $n_{\mathrm{S}}=1.5$. The ratio of the maximal amplitudes is found to be 1.10 and 1.23 , respectively. The refractive index of proteins is known to lie in the range of 1.45 and 1.65 [62], and the thickness of a CCMV capsid is $5 \mathrm{~nm}$ [63]. We expect thus to have similar effects on $_{304}$ experimental measurements.

Figure 2(b) shows the experimental extinction spec-306
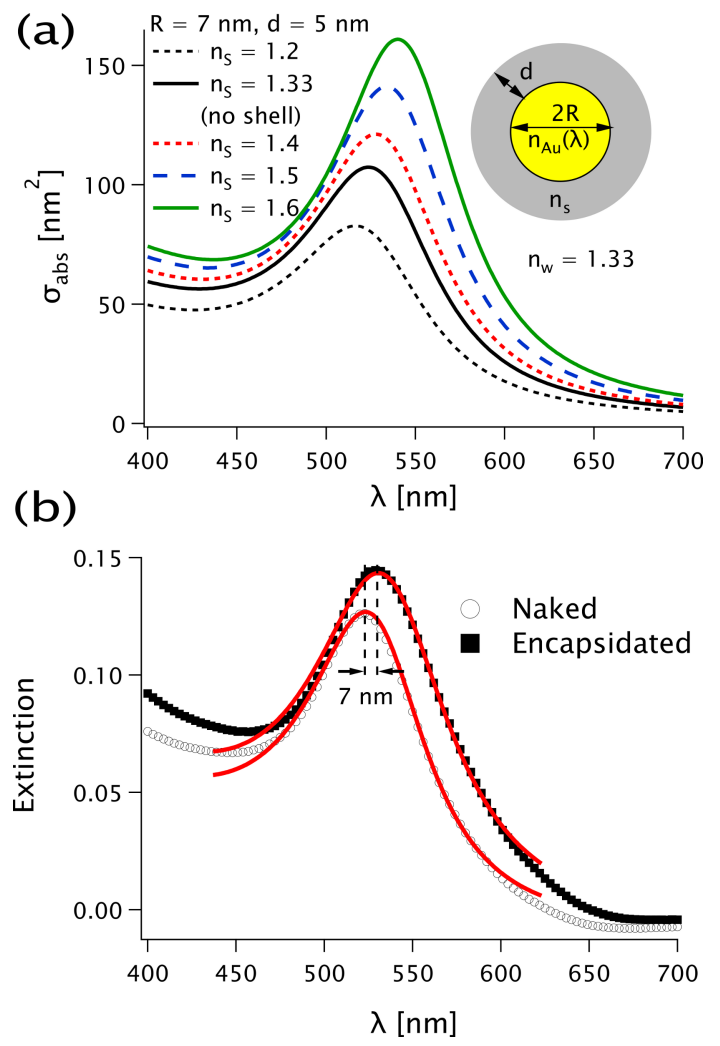

FIG. 2. UV-Vis absorbance spectroscopy of protein-coated AuNPs. (a) Calculated absorption cross-section of spherical AuNPs with a radius $R=7 \mathrm{~nm}$ and coated with a dielectric shell of thickness $d=5 \mathrm{~nm}$ with varying refractive indices $n_{\mathrm{S}}$. (b) Experimental extinction spectra of naked and proteincoated AuNPs stabilized with $\mathrm{EG}_{6}-\mathrm{COOH}$ ligands. The red solid lines are theoretical models fitting the measurements by using $R=7 \mathrm{~nm}$, and either no shell (for the naked AuNPs, $\bigcirc$ ) or a shell with $d=5 \mathrm{~nm}$ and $n_{\mathrm{S}}=1.44$ (for the encapsidated spheres,

tra measured on naked and protein-coated AuNPs. The spectrum of naked AuNPs is fitted with a model of gold spheres having a radius of $7 \mathrm{~nm}$. The spectrum of 
protein-coated AuNPs is fitted with the same model of ${ }_{337}$ gold spheres, but coated with a 5 -nm-thick protein layer, зз8 yielding a refractive index $n_{\mathrm{S}}=\sqrt{\epsilon_{\mathrm{S}}}=1.44$. The shift of 339 the resonance wavelength is found to be $7 \mathrm{~nm}$. The fit-340 ting values are in agreement with the expected thickness 341 and relative permittivity of a protein capsid.

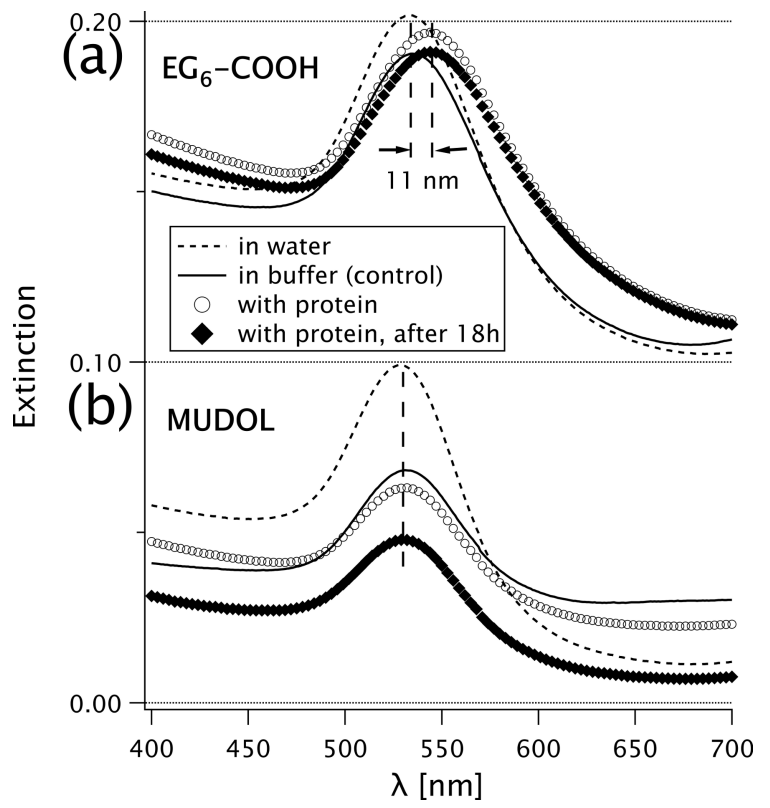

FIG. 3. Effect of ligands on the coating of AuNPs by proteins. Extinction spectra of AuNPs stabilized by (a) $\mathrm{EG}_{6}-\mathrm{COOH}$ and (b) MUDOL ligands in pure water (dashed line), in buffer solution (solid line), in buffer solution just after mixing with capsid proteins (open discs), and in buffer solution $18 \mathrm{~h}$ after mixing with capsid proteins (full diamonds).

Figures 3(a) and (b) show the effect of ligands on the coating of AuNPs by viral proteins. The extinction spectra of AuNPs in pure water and in buffer solution are similar for both ligands, there is therefore no immediate effect of the buffer solution on AuNPs. More importantly, the extinction of $\mathrm{EG}_{6}$ - $\mathrm{COOH}$-stabilized AuNPs in the presence of proteins exhibits a redshift of the resonance 345 wavelength of $9 \mathrm{~nm}$, whereas the resonance remains at 346 the same wavelength for MUDOL-stabilized AuNPs with ${ }_{347}$ proteins. This difference demonstrates that proteins are 348 adsorbed on $\mathrm{EG}_{6}-\mathrm{COOH}$-stabilized AuNPs but not on 349 MUDOL-stabilized AuNPs: The surface charge density 350 of AuNPs has to be negative and sufficiently high in ab-351 solute value to allow the adsorption of proteins, in $\operatorname{good}_{352}$ agreement with the findings reported by Daniel et al.353 [43]. The fact that the shift is $9 \mathrm{~nm}$ while it is $7 \mathrm{~nm}$ in 354 the previous measurements [Fig. 2(b)] may arise from a355 slightly larger average thickness of the protein shell due 356 to experimental variations in the subunit-to-AuNP molar 357 ratios. The extinction of MUDOL-stabilized AuNPs in 358 the presence of proteins after $18 \mathrm{~h}$ is significantly lower 359 than immediately after mixing [Fig. 3(b)], unlike the 360 case of $\mathrm{EG}_{6}-\mathrm{COOH}$-stabilized AuNPs in the presence of ${ }_{361}$ proteins [Fig. 3(a)]. Because MUDOL-stabilized AuNP\$362 have a neutral surface charge density and are not further stabilized by a layer of proteins, they massively aggregate due to strong attractive van der Waals forces and precipitate at the bottom of the tube. As a consequence, the concentration of AuNPs in the supernatant drops and so does the extinction.

\section{Adsorption dynamics of proteins on nanoparticles}

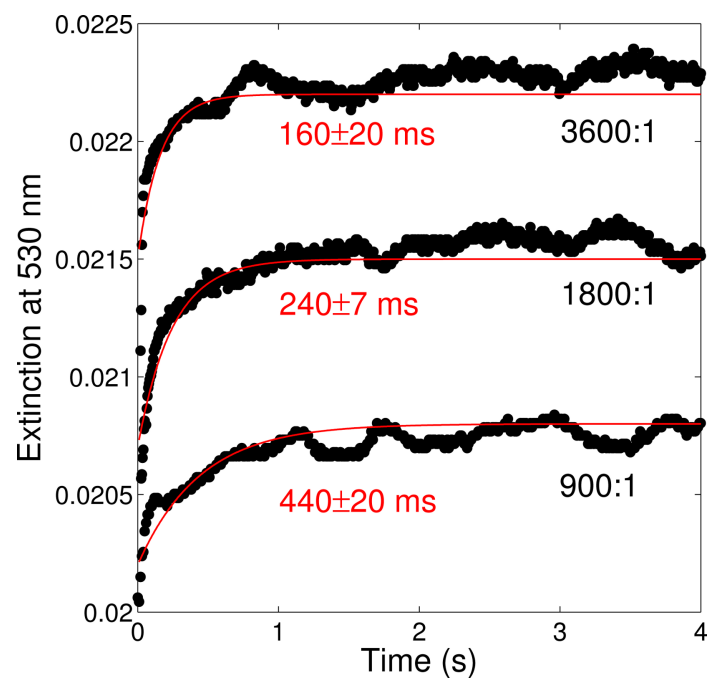

FIG. 4. Time-resolved extinction of protein-coated AuNPs, at various subunit-to-AuNP molar ratios. The red lines are exponential decay fits and the numbers in red are the corresponding decay times. The curves are shifted for clarity.

We investigate now the adsorption dynamics of proteins on $\mathrm{EG}_{6}-\mathrm{COOH}$-stabilized AuNPs in neutral buffer. Proteins and AuNPs are rapidly mixed and the extinction at $530 \mathrm{~nm}$ is monitored during the course of protein adsorption. As proteins are adsorbed on AuNPs, the wavelength of the plasmon resonance is gradually shifted from $520 \mathrm{~nm}$ to $530 \mathrm{~nm}$ in such a way that the extinction measured at $530 \mathrm{~nm}$ increases. Figure 4 gives the corresponding traces for three different molar ratios, with proteins in large excess. The adsorption occurs very rapidly, i.e., within a few hundreds of milliseconds. The adsorption timescale $\tau_{\text {ads }}$ decreases when the protein concentration $c_{\mathrm{S}}$ increases in such a way that $\tau_{\text {ads }} \propto c_{\mathrm{S}}{ }^{-0.8 \pm 0.2}$. A purely diffusion-limited process would yield $\tau_{\text {ads }} \propto c_{\mathrm{S}}{ }^{-1}$. The slight difference in the exponent may be due to a free energy barrier, most likely arising from the adsorbed subunits hindering the insertion of incoming free subunits through steric and electrostatic repulsions. 

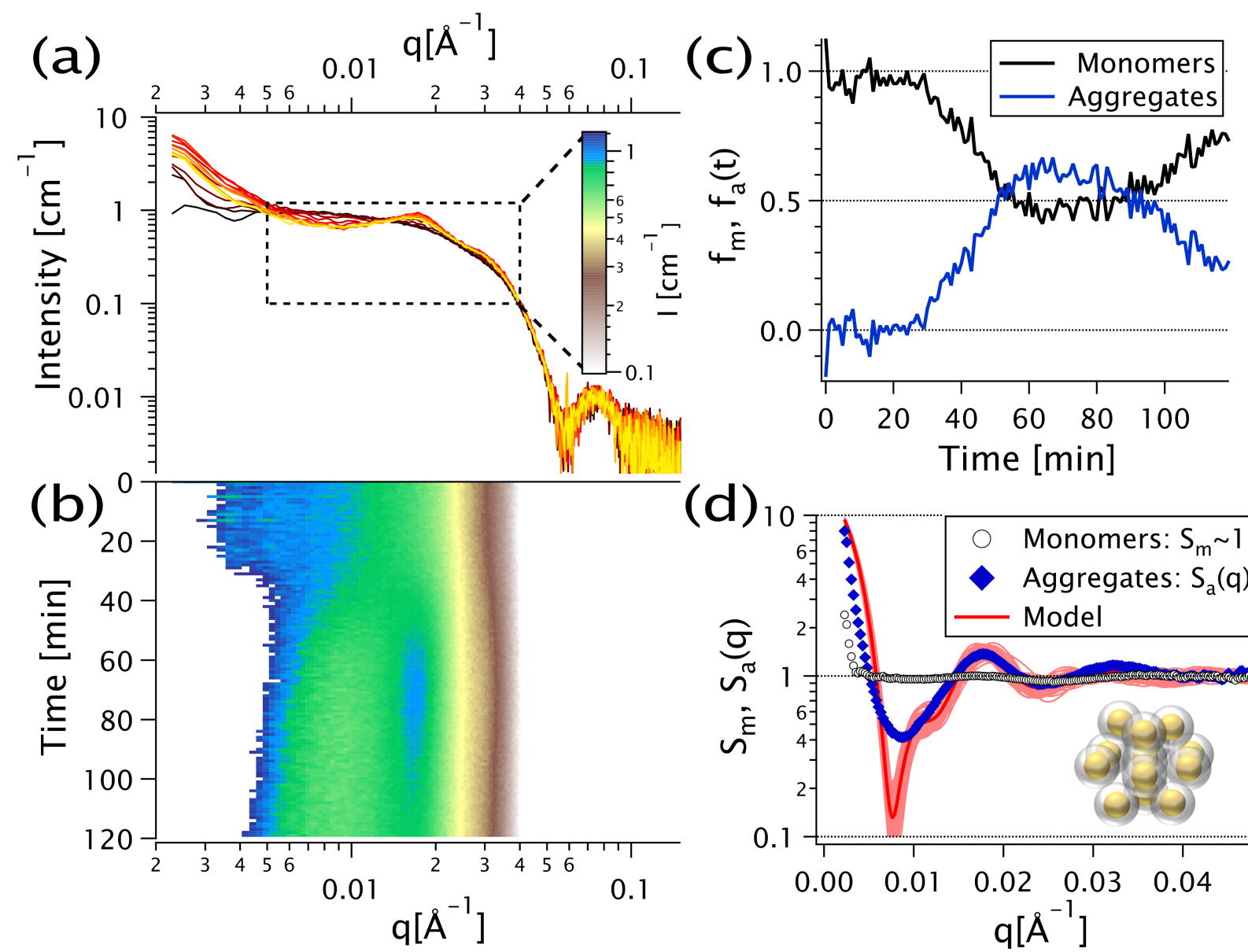

(d) 10

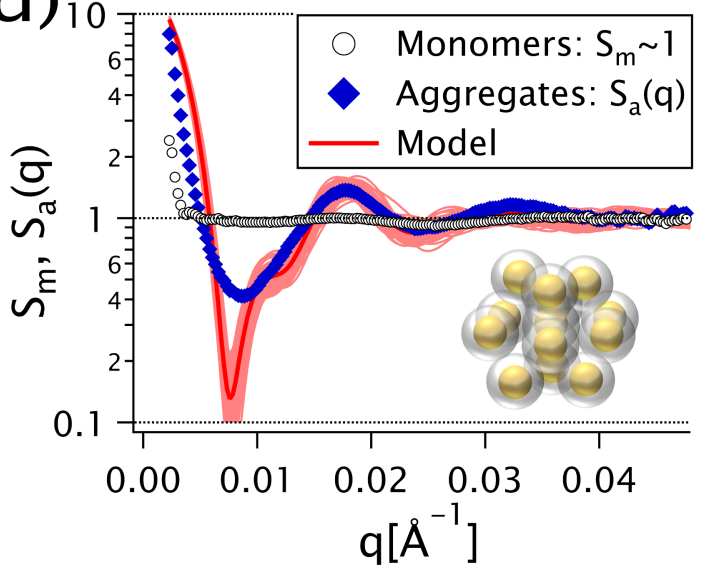

FIG. 5. Time-resolved small-angle X-ray scattering of $\mathrm{EG}_{6}-\mathrm{COOH}$-stabilized AuNPs coated by capsid proteins. (a) Scattering patterns collected at different time steps. (b) Two-dimensional representation of the scattering intensities as a function of time and wavenumber for the area delimited by the dashed box in (a). (c) Molar fractions of monomers (free protein-coated AuNPs) $f_{\mathrm{m}}$ and protein-coated AuNPs within aggregates $f_{\mathrm{a}}$ as a function of time inferred from singular value decomposition. (d) Corresponding structure factors of monomers $S_{\mathrm{m}}(q)$ and aggregates $S_{\mathrm{a}}(q)$. The light red lines are one hundred calculated structure factors of aggregates made of 13 particles separated by a distance fluctuating around $43 \mathrm{~nm}$ (3D model in inset) and the dark red line is their average. The protein concentration is 0.13 g. $\mathrm{L}^{-1}$ and the subunit-to-AuNP molar ratio is $2640: 1$.

\section{Assembly and dissociation dynamics of aggregates made of protein-coated AuNPs}

Next, we probe the large-scale structures formed by ${ }^{384}$ protein-coated AuNPs by using TR-SAXS with a high ${ }^{385}$ brilliance synchrotron source. Because the electron density of gold is considerably higher than that of proteins, the scattering intensity arising from capsid proteins is negligible with respect to that due to AuNPs. However, if the protein-coated AuNPs transiently form aggregates ${ }_{386}$ as reported by Malyutin and Dragnea [30], the presence 387 of the latter can be readily detected via the modulation $_{388}$ they induce in the structure factor.

Figure 5(a) shows the apparition of a peak at $q \simeq 0.018_{390}$ $\AA^{-1}$, due to loosely-packed AuNPs aggregates. The two-391 dimensional representation of Fig. 5(b) reveals that the 392 peak (visible as a blue streak) appears about $50 \mathrm{~min}$ af- -393 ter mixing subunits and AuNPs, and disappears almost 394 completely after $110 \mathrm{~min}$. The isosbestic points on the 395 scattering intensities of Fig. 5(a) at $q \simeq 5.5 \times 10^{-3}$ and $0.013 \AA^{-1}$ suggest the presence of only two species: free (monomers) and aggregated protein-coated AuNPs, interconverting over the course of the process, in which case the scattering intensity $I(q, t)$ reduces to [64]:

$$
I(q, t) \propto\left[f_{\mathrm{m}}(t)+f_{\mathrm{a}}(t) S_{\mathrm{a}}(q)\right] P(q)
$$

where $f_{\mathrm{m}}$ and $f_{\mathrm{a}}$ stand for the molar fractions of free protein-coated AuNPs and protein-coated AuNPs within aggregates, respectively. $S_{\mathrm{a}}(q)$ is the structure factor of the aggregates and $P(q)$ is the form factor of AuNPs. $S_{\mathrm{a}}(q)$ must be seen as a structure factor averaged over all aggregates, during the whole process. $P(q)$ is obtained from the first scattering pattern, when proteincoated AuNPs are still all free in solution. The molar fractions and $S_{\mathrm{a}}(q)$ are estimated by retaining the two first components of a singular value decomposition [65] 
performed on $I(q) / P(q)$. By rotating and rescaling the 448 basis vectors in order to reproduce the flat structure fac-449 tor of monomers, i.e., $S_{\mathrm{m}}(q) \sim 1, f_{\mathrm{m}}(t)$ can be estimated.450 $f_{\mathrm{a}}(t)$ and $S_{\mathrm{a}}(q)$ are then deduced by mass conservation. 45 $f_{\mathrm{m}}(t)$ and $f_{\mathrm{a}}(t)$ are plotted in Fig. 5(c). The first 452 aggregates appear about 30 min after mixing and their 453 fraction continues to grow until $t \simeq 60 \mathrm{~min}$. At this454 moment, around $60 \%$ of AuNPs are within aggregates.455 Then, AuNPs are slowly released but $2 \mathrm{~h}$ after mixing, 456 there are still $f_{\mathrm{a}}=25 \%$ in aggregated form.

E. Modeling the structure factor of the aggregates 460

The structure factor of the aggregates $S_{\mathrm{a}}(q)$ is shown in ${ }^{462}$ Fig. 5(d). Since all interactions are presumably isotropic, ${ }^{463}$ we describe it based on a compact reference assembly ${ }^{464}$ comprising 13 particles, one in the center and the re-465 maining twelve forming a shell around it. The distance ${ }^{460}$ between nearest neighbors is initially $43 \mathrm{~nm}$, but the par- ${ }^{467}$ ticles are then allowed to fluctuate at random around $_{468}$ their equilibrium positions according to Gaussian distri- ${ }_{469}$ butions with standard deviation $6.45 \mathrm{~nm}$ along each space ${ }_{470}$ dimension $(x, y, z)$. The structure factors corresponding ${ }_{471}$ to one hundred noise realizations are shown in light red, ${ }_{472}$ and their average in dark red.

Despite its simplicity, the model is in qualitative agree- ${ }_{474}$ ment with the experimental data: the soft peak at $0.018_{475}$ $\AA^{-1}$, indicative of a limited degree of order (as for $\mathrm{an}_{476}$ amorphous packing) is well captured. The discrepancies: ${ }_{477}$ a sharp minimum at $0.0076 \AA^{-1}$ and a rounded increase A78 at lower $q$ values, can be explained by the size polydisper- ${ }_{479}$ sity of the aggregates in solution. Note that the scattering signal is largely dominated by the gold nanoparticles and provides no direct information on the position and conformation of the proteins.

Given that the diameter of AuNPs is $15 \mathrm{~nm}$ as inferred from $P(q), \mathrm{EG}_{6}-\mathrm{COOH}$ ligand is $2 \mathrm{~nm}$ long at most, and ${ }^{480}$ the thickness of a CCMV capsid is $5 \mathrm{~nm}$, the full diameter of protein-coated AuNPs is estimated to be $29 \mathrm{~nm}$. $\operatorname{In}_{481}$ other words, the surfaces of protein-coated AuNPs within ${ }_{482}$ an aggregate are separated by a gap of $15 \mathrm{~nm}$, most likely $y_{483}$ filled with capsid proteins. In another experiment where ${ }_{484}$ the subunit-to-AuNP molar ratio is reduced by a factor ${ }_{485}$ of two while keeping the subunit concentration identical ${ }_{486}$ the structure peak is barely visible and there is almost ${ }_{487}$ no evolution of the scattering patterns. It suggests that ${ }_{488}$ the subunit-to-AuNP molar ratio as well as the subunit ${ }_{489}$ concentration have to be sufficiently high to induce the formation of transient aggregates.

By combining UV-Vis absorbance spectroscopy and TR-SAXS, we probe the self-assembly of CCMV capsids templated by ligand-stabilized AuNPs with different 492 temporal and spatial scales. The measurements allow493 us to propose a three-step pathway: Right after mixing proteins and ligand-stabilized AuNPs, the proteins, driven by electrostatic interactions, adsorb on the AuNPs provided that the surface charge density is negative and high enough in absolute value. The adsorption occurs on the subsecond timescale and is accelerated when the subunit-to-AuNP molar ratio increases. In a large excess of subunits, a part of the protein-coated AuNPs form aggregates in coexistence with the remaining free proteincoated AuNPs within the first hour. The surface-tosurface distance between protein-coated AuNPs within an aggregate is sufficiently large to accommodate two or three layers of capsid proteins, and each aggregate comprises a dozen or so nanoparticles. Notice that the aggregates are soluble and do not precipitate at the bottom of the tube. Then, the aggregates slowly dissociate over a couple of hours by releasing individual protein-coated AuNPs. The latter are stable over long durations and electron microscopy images reveal a closed shell wrapping up each AuNP.

There is a need to better understand and subsequently, control the nonequilibrium self-assembly and self-organization dynamics of complex molecular systems [66]. Biological systems, spurred by the pressure of their own survival, have evolved to spontaneously build up large-scale, perfectly ordered structures in an errorfree manner, despite an often adverse environment. The present work highlights the robustness and adaptability of viral proteins during the packaging of a cargo, as it efficiently occurs in host cell. It should promote further fundamental studies on bio-inspired systems, notably related to the physics of dynamical phenomena.

\section{ACKNOWLEDGMENTS}

M.C. is supported by the "IDI 2016" project funded by the IDEX Paris-Saclay, ANR-11-IDEX-0003-02. G.T. acknowledges financial support from the Agence Nationale de la Recherche (contract ANR-16-CE30-0017-01). We also acknowledge the SOLEIL synchrotron for allocation of synchrotron beam time on the SWING beamline. The electron microscopy imaging is supported by "Investissements d'Avenir" LabEx PALM (ANR-10-LABX0039-PALM).

\section{Appendix: Analytical model of the dielectric function of gold}

The relative permittivity of gold is approximated by the analytical model used by Etchegoin et al. [67]: 


$$
\begin{aligned}
\epsilon_{\mathrm{Au}}= & \epsilon_{\infty}-\frac{1}{\lambda_{\mathrm{p}}{ }^{2}\left(\frac{1}{\lambda^{2}}+\frac{\mathrm{i}}{\gamma_{\mathrm{p}} \lambda}\right)} \\
& +\sum_{i=1,2} \frac{A_{i}}{\lambda_{i}}\left[\frac{\mathrm{e}^{\mathrm{i} \phi_{i}}}{\frac{1}{\lambda_{i}}-\frac{1}{\lambda}-\frac{1}{\gamma_{i}}}+\frac{\mathrm{e}^{-\mathrm{i} \phi_{i}}}{\frac{1}{\lambda_{i}}+\frac{1}{\lambda}+\frac{1}{\gamma_{i}}}\right]
\end{aligned}
$$

The first two terms come from the Drude model while $e^{505}$ the summation is over two terms which come from inter-506 band transitions. $\lambda_{\mathrm{p}}$ is the plasma wavelength, $\gamma_{\mathrm{p}}$ is the
[1] Q. Luo, C. Hou, Y. Bai, R. Wang, and J. Liu, Chem.554 Rev. 116, 13571 (2016).

[2] W. H. Roos, R. Bruinsma, and G. J. L. Wuite, Nat.556 Phys. 6, 733 (2010).

[3] M. G. Mateu, Arch. Biochem. Biophys. 531, 65 (2013). 558

[4] G. Tresset, V. Decouche, J.-F. Bryche, A. Charpilienne,559 C. Le Coeur, C. Barbier, G. Squires, M. Zeghal, D. Pon-560 cet, and S. Bressanelli, Arch. Biochem. Biophys. 537,561 144 (2013).

[5] M. F. Hagan, Adv. Chem. Phys. 155, 1 (2014).

[6] R. F. Bruinsma and W. S. Klug, Annu. Rev. Condens.564 Matter Phys. 6, 245 (2015).

[7] J. D. Perlmutter and M. F. Hagan, Annu. Rev. Phys.566 Chem. 66, 217 (2015).

[8] T. Verdier, L. Foret, and M. Castelnovo, J. Phys. Chem.568 B 120, 6411 (2016).

[9] R. Zandi and P. van der Schoot, Biophys. J. 96, 9 (2009).570

[10] M. Comas-Garcia, R. D. Cadena-Nava, A. L. N. Rao,571 C. M. Knobler, and W. M. Gelbart, J. Virol. 86, 12271572 (2012).

[11] A. Borodavka, R. Tuma, and P. G. Stockley, Proc. Natl.574 Acad. Sci. U. S. A. 109, 15769 (2012).

12] M. Castelnovo, D. Muriaux, and C. Faivre-Moskalenko,56 New J. Phys. 15, 035028 (2013).

[13] A. Zlotnick, J. Z. Porterfield, and J. C.-Y. Wang, Bio-578 phys. J. 104, 1595 (2013).

14] R. F. Garmann, M. Comas-Garcia, A. Gopal, C. M. Kno-58 bler, and W. M. Gelbart, J. Mol. Biol. 426, 1050 (2014).581

[15] N. Patel, E. C. Dykeman, R. H. A. Coutts, G. P.582 Lomonossoff, D. J. Rowlands, S. E. V. Phillips, N. Ran-583 son, R. Twarock, R. Tuma, and P. G. Stockley, Proc.584 Natl. Acad. Sci. U. S. A. 112, 2227 (2015).

585

[16] R. F. Bruinsma, M. Comas-Garcia, R. F. Garmann, and 586 A. Y. Grosberg, Phys. Rev. E Stat. Nonlin. Soft Matter 587 Phys. 93, 032405 (2016).

588

[17] R. F. Garmann, M. Comas-Garcia, C. M. Knobler, and 589 W. M. Gelbart, Acc. Chem. Res. 49, 48 (2016).

[18] S. Li, G. Erdemci-Tandogan, P. van der Schoot, and591 R. Zandi, J. Phys. Condens. Matter 30, 044002 (2018). 592

[19] F. D. Sikkema, M. Comellas-Aragons, R. G. Fokkink,593 B. J. M. Verduin, J. J. L. M. Cornelissen, and R. J. M.594 Nolte, Org. Biomol. Chem. 5, 54 (2007).

595 20] Y. Hu, R. Zandi, A. Anavitarte, C. M. Knobler, and 596 W. M. Gelbart, Biophys. J. 94, 1428 (2008). 597

[21] O. M. Elrad and M. F. Hagan, Phys. Biol. 7, 045003598 (2010).

[22] M. Comellas-Aragones, F. D. Sikkema, G. Delaittre, 600 damping expressed as a wavelength, $A_{i}$ is the amplitude of each term, $\lambda_{i}$ and $\gamma_{i}$ are the wavelength and damping of the respective interband transitions. $\phi_{i}$ is the phase of each transition. The parameter values correspond to the Johnson and Christy data [68], with the exception of $\lambda_{1}$, which we modify slightly to obtain better agreement with the spectrum of the naked particles: $\epsilon_{\infty}=1.54, \lambda_{\mathrm{p}}=143$ $\mathrm{nm}, \gamma_{\mathrm{p}}=14500 \mathrm{~nm}, A_{1}=1.27, \phi_{1}=-\pi / 4, \lambda_{1}=445$ $\mathrm{nm}$ (470 nm for Johnson and Christy), $\gamma_{1}=1900 \mathrm{~nm}$, $A_{2}=1.1, \phi_{2}=-\pi / 4, \lambda_{2}=325 \mathrm{~nm}$ and $\gamma_{2}=1060 \mathrm{~nm}$.
A. E. Terry, S. M. King, D. Visser, R. K. Heenan, R. J. M. Nolte, J. J. L. M. Cornelissen, and M. C. Feiters, Soft Matter 7, 11380 (2011).

[23] R. D. Cadena-Nava, Y. Hu, R. F. Garmann, B. Ng, A. N. Zelikin, C. M. Knobler, and W. M. Gelbart, J. Phys. Chem. B 115, 2386 (2011).

[24] G. Tresset, M. Tatou, C. Le Coeur, M. Zeghal, V. Bailleux, A. Lecchi, K. Brach, M. Klekotko, and L. Porcar, Phys. Rev. Lett. 113, 128305 (2014).

[25] J. Sun, C. DuFort, M.-C. Daniel, A. Murali, C. Chen, K. Gopinath, B. Stein, M. De, V. M. Rotello, A. Holzenburg, C. C. Kao, and B. Dragnea, Proc Natl Acad Sci U S A 104, 1354 (2007).

[26] N. L. Goicochea, M. De, V. M. Rotello, S. Mukhopadhyay, and B. Dragnea, Nano Lett. 7, 2281 (2007).

[27] S. E. Aniagyei, C. J. Kennedy, B. Stein, D. A. Willits, T. Douglas, M. J. Young, M. De, V. M. Rotello, D. Srisathiyanarayanan, C. C. Kao, and B. Dragnea, Nano Lett. 9, 393 (2009).

[28] C. C. DuFort and B. Dragnea, Annu. Rev. Phys. Chem. 61, 323 (2010).

[29] L. He, Z. Porterfield, P. van der Schoot, A. Zlotnick, and B. Dragnea, ACS Nano 7, 8447 (2013).

[30] A. G. Malyutin and B. Dragnea, J. Phys. Chem. B 117, 10730 (2013).

[31] R. Kusters, H.-K. Lin, R. Zandi, I. Tsvetkova, B. Dragnea, and P. van der Schoot, J. Phys. Chem. B 119, 1869 (2015).

[32] A. Liu, M. Verwegen, M. V. de Ruiter, S. J. Maassen, C. H.-H. Traulsen, and J. J. L. M. Cornelissen, J. Phys. Chem. B 120, 6352 (2016).

[33] Z. Ye, L. Wei, X. Zeng, R. Weng, X. Shi, N. Wang, L. Chen, and L. Xiao, Anal. Chem. 90, 1177 (2018).

[34] E. C. Dreaden, A. M. Alkilany, X. Huang, C. J. Murphy, and M. A. El-Sayed, Chem. Soc. Rev. 41, 2740 (2012).

[35] M. Daniel and D. Astruc, Chem. Rev. 104, 293 (2004).

[36] V. Myroshnychenko, J. Rodriguez-Fernandez, I. Pastoriza-Santos, A. M. Funston, C. Novo, P. Mulvaney, L. M. Liz-Marzan, and F. J. Garcia de Abajo, Chem. Soc. Rev. 37, 1792 (2008).

[37] R. Zandi, D. Reguera, R. F. Bruinsma, W. M. Gelbart, and J. Rudnick, Proc. Natl. Acad. Sci. U. S. A. 101, 15556 (2004)

[38] V. L. Lorman and S. B. Rochal, Phys. Rev. Lett. 98, 185502 (2007).

[39] S. B. Rochal, O. V. Konevtsova, A. E. Myasnikova, and V. L. Lorman, Nanoscale 8, 16976 (2016). 
[40] W. K. Kegel and P. van der Schoot, Biophys. J. 86, 3905633 (2004).

[41] J. Chen, M. Chevreuil, S. Combet, Y. Lansac, and 635 G. Tresset, J. Phys. Condens. Matter 29, 474001 (2017).636

[42] G. Tresset, J. Chen, M. Chevreuil, N. Nhiri, E. Jacquet,637 and Y. Lansac, Phys. Rev. Applied 7, 014005 (2017). ${ }_{638}$

[43] M.-C. Daniel, I. B. Tsvetkova, Z. T. Quinkert, A. Murali,639 M. De, V. M. Rotello, C. C. Kao, and B. Dragnea, ACS 640 Nano 4, 3853 (2010).

44] J. M. Johnson, J. Tang, Y. Nyame, D. Willits, M. J.642 Young, and A. Zlotnick, Nano Lett. 5, 765 (2005). 643

[45] C. Chen, C. C. Kao, and B. Dragnea, J. Phys. Chem. A644 112, 9405 (2008).

[46] Z. D. Harms, L. Selzer, A. Zlotnick, and S. C. Jacobson, 646 ACS Nano 9, 9087 (2015).

[47] M. Castellanos, R. Pérez, P. J. P. Carrillo, P. J. de Pablo,648 and M. G. Mateu, Biophys. J. 102, 2615 (2012). 649

[48] M. Medrano, M. A. Fuertes, A. Valbuena, P. J. P. Car-650 rillo, A. Rodriguez-Huete, and M. G. Mateu, J. Am.651 Chem. Soc. 138, 15385 (2016).

[49] S. Milles, M. R. Jensen, G. Communie, D. Maurin,653 G. Schoehn, R. W. H. Ruigrok, and M. Blackledge, 654 Angew. Chem. Int. Ed. 55, 9356 (2016).

[50] C. A. Lutomski, N. A. Lyktey, E. E. Pierson, Z. Zhao, 656 A. Zlotnick, and M. F. Jarrold, J. Am. Chem. Soc. 140,657 5784 (2018).

51] S. Kler, R. Asor, C. Li, A. Ginsburg, D. Harries, A. Op-659 penheim, A. Zlotnick, and U. Raviv, J. Am. Chem. Soc.660 134, 8823 (2012).

[52] G. Tresset, C. Le Coeur, J.-F. Bryche, M. Tatou,662 M. Zeghal, A. Charpilienne, D. Poncet, D. Constantin, 663 and S. Bressanelli, J. Am. Chem. Soc. 135, 15373 (2013).664
[53] D. Law-Hine, A. K. Sahoo, V. Bailleux, M. Zeghal, S. Prevost, P. K. Maiti, S. Bressanelli, D. Constantin, and G. Tresset, J. Phys. Chem. Lett. 6, 3471 (2015).

[54] D. Law-Hine, M. Zeghal, S. Bressanelli, D. Constantin, and G. Tresset, Soft Matter 12, 6728 (2016).

[55] M. Chevreuil, D. Law-Hine, J. Chen, S. Bressanelli, S. Combet, D. Constantin, J. Degrouard, J. Möller, M. Zeghal, and G. Tresset, Nat. Commun. 9, 3071 (2018).

[56] A. Ali and M. J. Roossinck, J. Virol. Methods 141, 84 (2007).

[57] H. Hiramatsu and F. E. Osterloh, Chem. Mater. 16, 2509 (2004).

[58] X. Ye, L. Jin, H. Caglayan, J. Chen, G. Xing, C. Zheng, V. Doan-Nguyen, Y. Kang, N. Engheta, C. R. Kagan, and C. B. Murray, ACS Nano 6, 2804 (2012).

[59] J. Piella, N. G. Bastús, and V. Puntes, Chem. Mater. 28, 1066 (2016).

[60] C. Bohren and D. R. Huffman, Absorption and scattering of light by small particles (Wiley-VCH, New York, 2008).

[61] K. Slyusarenko, B. Abécassis, P. Davidson, and D. Constantin, Nanoscale 6, 13527 (2014).

[62] D. B. Hand, J. Biol. Chem. 108, 703 (1935).

[63] J. A. Speir, S. Munshi, G. Wang, T. S. Baker, and J. E. Johnson, Structure 3, 63 (1995).

[64] R. J. Hunter, Foundations of colloid science (Oxford University Press, New York, 2001).

[65] E. R. Henry, Biophys. J. 72, 652 (1997).

[66] S. Whitelam and R. L. Jack, Annu. Rev. Phys. Chem. Annual Review of Physical Chemistry, 66, 143 (2015).

[67] P. G. Etchegoin, E. C. Le Ru, and M. Meyer, J. Chem. Phys. 125, 164705 (2006).

[68] P. Johnson and R. Christy, Phys. Rev. B 6, 4370 (1972). 